\title{
eJRIEPS
}

Ejournal de la recherche sur l'intervention en éducation physique et sport

Hors-série $N^{\circ} 1 \mid 2015$

Quelques aspects théoriques de la didactique des sports collectifs. Préparation aux concours de recrutement

\section{Enseigner, observer et évaluer l'efficacité collective des élèves en football}

\section{Guillaume Dietsch et Jean-Francis Gréhaigne}

\section{OpenEdition}

Journals

Édition électronique

URL : http://journals.openedition.org/ejrieps/1316

DOI : $10.4000 /$ ejrieps. 1316

ISSN : 2105-0821

Éditeur

ELLIADD

Référence électronique

Guillaume Dietsch et Jean-Francis Gréhaigne, «Enseigner, observer et évaluer l'efficacité collective des élèves en football », eJRIEPS [En ligne], Hors-série N 1 | 2015, mis en ligne le 01 décembre 2015, consulté le 03 octobre 2019. URL : http://journals.openedition.org/ejrieps/1316 ; DOI : 10.4000/ ejrieps. 1316 
eJRIEPS Hors série n¹ Décembre 2015

Enseigner, observer et évaluer l'efficacité collective des élèves en football

Guillaume Dietsch* \& Jean-Francis Gréhaigne **

*Professeur Agrégé d'EPS, Lycée Professionnel Aristide Briand, Le Blanc-Mesnil

Doctorant au LIRTES (EA7313), Université Paris-Est

** Professeur des Universités retraité, Université Bourgogne Franche Comté

Historiquement, l'enseignement $d u$ football à l'école est sujet à des perceptions paradoxales (Grün, 2012). Au regard de sa représentativité culturelle et de sa diffusion massive dans la société, le football devrait occuper une place prépondérante dans les programmations des APSA scolaires. Or, des obstacles s'opposent à une expansion du football à l'école. Les représentations parfois négatives qu'il véhicule l'empêchent d'asseoir sa place à l'école, notamment auprès des enseignants d'Education Physique et Sportive (EPS). Les réticences de ces derniers à proposer l'activité football semblent renforcées en milieu difficile " citadin " au regard du rapport à l'activité des élèves. En effet, les élèves de "quartier » privilégient «l'exploit individuel » (Travert, 1997) au détriment d'une efficacité collective. Ce type de joueur " perso » (Caillé, 2008) se singularise en réalisant l'exploit et en effectuant de beaux gestes mais perd souvent le ballon. De plus, le rapport à la règle de ces élèves et son acceptation est problématique, ce qui ne favorise pas le bon déroulement du jeu (Marle \& Duprat, 2014). Or actuellement, l'émergence du futsal, qui amène une transformation de la pratique du football dans les " quartiers ", vise à dépasser ces tensions grâce à des règles bien plus strictes qu'en football comme la règle des « fautes collectives » (Dietsch, 2015).

Un autre point constitue un obstacle important à l'enseignement du football. En effet, le modèle d'entraînement de la pratique sociale de référence est survalorisé par certains élèves. Dans les cours d'EPS, le modèle technique est fortement ancré dans l'imaginaire des garçons, au point de constituer un noyau dur et stable de représentations. II est à noter, également, que l'on assiste dans le cadre de l'enseignement des sports collectifs à une survalorisation des savoirs pratiques tels que définit dans la littérature technique.

Enfin, en plus de ces obstacles à enseigner le football à l'école se surajoutent des difficultés pour les enseignants à évaluer ce sport collectif, notamment lorsqu'on leur demande d'observer la "pertinence et l'efficacité de l'organisation collective » (MEN, $2009 ; 2010)$. Nous allons y revenir. 


\section{eJRIEPS Hors série n¹ Décembre 2015}

A cet effet, nous proposons dans cet article des outils d'analyse, d'observation et d'évaluation de la motricité des élèves en relation avec les savoirs tactiques collectifs.

\section{Observer et évaluer les savoirs collectifs des élèves en football}

Toute situation d'évaluation ou d'observation en sports collectifs repose sur un certain nombre de présupposés qui ont évolué dans le temps en fonction des conceptions en vogue. On est passé du jeu global, aux parcours chronométrés, (...) pour en arriver à des situations aménagées en jeu réduit. Les outils ont été successivement "l'oeil du maquignon", des fiches d'observation quantitatives de l'efficacité individuelle ou collective, le chronomètre, des grilles de comportements de plus en plus sophistiquées et de plus en plus difficiles à utiliser, pour en arriver maintenant à tenter de combiner des aspects quantitatifs et qualitatifs avec les indices numériques ou le nombre d'actions positives. L'évolution de ces outils et de leur utilisation souligne la difficulté d'évaluer en sport collectif, en particulier quand on met en avant la notion d'opposition. En effet, si elle est novatrice du point de vue de la conception de l'apprentissage, ce type d'évaluation pose de réels problèmes pour l'évaluation des acquis. En effet, quelle est la part de chacun dans le succès ou l'échec de l'équipe ? Comment comparer deux équipes qui ne s'affrontent pas? Quels indicateurs fiables pour évaluer un rapport de force? Autant de questions nouvelles auxquelles il nous faut tenter de répondre.

\section{1. Description / observation du jeu}

Une représentation graphique de données d'observation bien lisibles pour certaines classes est présentée figure 1 .

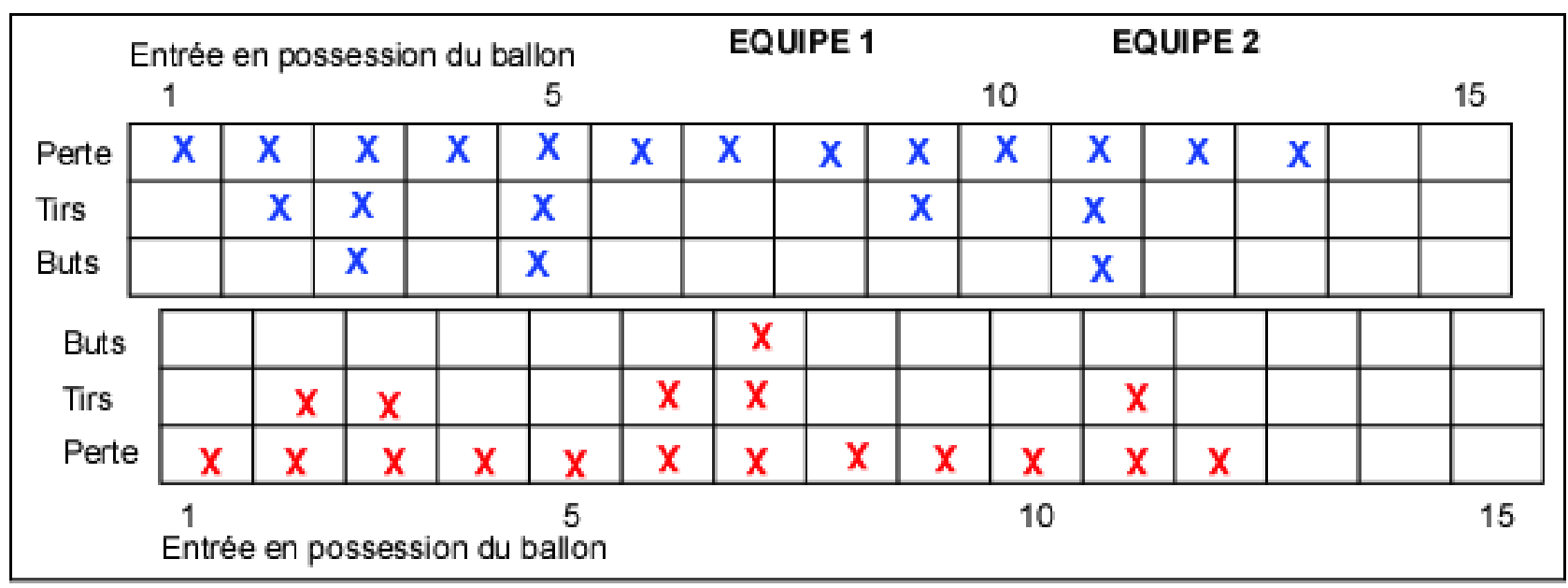

Figure 1. Représentation graphique : un visuel du score, des tirs, des pertes de balle, et du nombre d'attaque 


\section{eJRIEPS Hors série n¹ Décembre 2015}

Le simple relevé du nombre de buts marqués $(B)$, du nombre de tirs cadrés $(T)$ et du nombre d'entrées en possession du ballon $(E)$ dans des rencontres de durée constante et dans des conditions identiques, donne des indications très précieuses.

On peut, ainsi, calculer le nombre de balles conquises par unité de temps dans un rapport de forces équilibré. II semble que cela puisse être un indicateur significatif de niveaux de jeu. Car, plus l'échange de ballon entre les deux équipes est élevé plus les équipes sont faibles ou éventuellement de très bons niveaux. Cela permet de voir quand les buts sont marqués au début à la fin de la rencontre ou régulièrement tout au long du jeu. D'un autre point de vue, on peut mettre en rapport deux par deux les critères retenus :

Nombre de tirs cadrés $(T)$ / Nombre de balles conquises $(E)$

Nombre de buts marqués $(B)$ / Nombre de tentatives de tirs $(T)$

On obtient ainsi des informations objectives sous la forme d'un coefficient variant entre 0 et 1 qui permettent de caractériser le jeu par exemple lors d'une évaluation de départ ou de le réguler les actions en projet par un retour d'information efficace. Les applications sur le terrain de ce type de travail permettent de penser que l'on devrait pouvoir déterminer des constantes avec une faible dispersion. Ainsi pour les deux rapports évoqués plus haut, la norme dans une situation d'apprentissage en football en $4 \times 4$ et 7 minutes de jeu pourrait être de : $N$ pour le nombre de balles conquises ; N/2 pour le nombre de tentatives de tirs ; N/4 pour le nombre de buts marqués, les coefficients $T / E$ et $B / T$ tendraient alors vers 0,5 .

Un autre type d'outil un peu plus précis consiste en une fiche de repérage de la circulation du ballon (Dugrand, 1989). II consiste à relever les numéros des possesseurs successifs du ballon depuis la prise de balle jusqu'à sa perte (perte de balle, tir, but).

$4 ; 3 ; 5 ; 4 ; 3 ; 4$; Perte de balle (P.).

$4 ; 6 ; 3 ; \mathrm{P}$.

$5 ; 2 ; 4 ; 5$; Tirs.

$3 ; 2 ; 3$; Touche ; 5 ;P.

etc.

Ce type de recueil permet de repérer les statuts dominants dans une équipe et, en le corrélant avec d'autres données de préciser certains aspects du jeu. On peut également analyser pour chaque joueur, le rapport ballons reçus / ballons donnés. En effet dans une équipe :

- certains joueurs distribuent moins de ballons qu'ils n'en ont reçus ;

- d'autres équilibrent réception et distribution ; 


\section{eJRIEPS Hors série n¹ Décembre 2015}

- d'autres enfin distribuent plus de ballons qu'ils n'en reçoivent en particulier en cas de récupération active de la balle.

L'interprétation est souvent simple (bon joueur, joueur débutant, etc.) mais, parfois, ce constat est à combiner avec d'autres outils plus qualitatifs pour affiner l'interprétation d'une hypothèse liées aux seules données chiffrées.

Tableau I. Des exemples d'actions positives.

\begin{tabular}{|l|l}
\hline Pour les joueurs en phase offensive & Pour les joueurs en phase défensive \\
\hline Tirs cadrés, but marqué & Interception volontaire \\
Franchir la ligne d'avantage & Duel gagné \\
Réalisation d'un $1-2$ & Replacement entre le ballon et sa propre \\
Passe longue réussie à un partenaire en & cible \\
avant & Ecarter le ballon sans le récupérer \\
Passe décisive, centre en retrait & Recul fuite efficace. \\
Déviation sans contrôle vers l'avant & Gêner le porteur du ballon, flotter devant le \\
& porteur de balle
\end{tabular}

A cet effet, on peut avoir recours aux actions positives parfois ramenées à la minute de jeu pour faciliter les comparaisons car elles constituent un outil d'observation très efficace pour évaluer les actions souvent utilisées et la qualité du jeu. Les actions positives sont des actions de jeu profitables en fonction du but du jeu et constituent les conduites nouvelles attendues dans les configurations de jeu vécues. Quelques exemples d'actions significatives sont définis et présentés dans le tableau I. En fonction du contexte, de la situation de jeu et du niveau des joueurs, trois actions positives (AP) seront choisies pour l'observation.

Les études menées montrent que pour cinq minutes de jeu, un rapport de 0.7 ou 0.8 atteste d'une transformation dans les comportements des élèves. D'un point de vue pratique, l'enseignant peut gérer l'observation des différents joueurs (jusqu'au $5 \times 5$ ) avec l'aide d'un secrétaire. La focalisation essentiellement sur le porteur de balle ne pose guère de problème ; l'utilisation de jeux réduits, sur terrains adaptés, permet à tous les joueurs d'entrer très souvent en contact avec le ballon. II est à noter que les résultats de ces types d'observation peuvent être transmis immédiatement aux élèves et donner lieu à une notation si nécessaire (Marle, Zerai, \& Gréhaigne, 2011).

1. 2. Programme et évaluation du jeu collectif

En EPS, il convient aussi d'évaluer les apprentissages des élèves à partir d'indicateurs de progrès lisibles et pertinents. Toutefois, pour les enseignants, cette démarche 


\section{eJRIEPS Hors série n¹ Décembre 2015}

d'apprentissage semble souvent difficile à mettre en œuvre en sport collectif quand il faut caractériser et évaluer la «pertinence et l'efficacité de l'organisation collective » (MEN, 2009 ; 2010). Dans ce cadre, deux notions se trouvent au cœur de l'analyse du jeu collectif : d'une part, celle de configuration de jeu, telle qu'elle s'actualise et prend forme dans le rapport de force en cours, d'autre part, celle de situations potentiellement émergeantes, mais prédictibles à qui sait « lire » le jeu, et dont les joueurs doivent tirer parti (Gréhaigne, Caty \& Marle, 2004). Ces situations «émergeantes » sont également appelées «configurations prototypiques » par Gréhaigne (2007) et permettent une analyse qualitative du jeu en sport collectif.

Les enjeux de savoirs associés à la lecture du jeu renvoient à la compétence propre $n^{\circ} 4$ (« Conduire et maîtriser un affrontement collectif ») et relèvent non seulement de la dimension tactique («Pertinence et efficacité de l'organisation collective») mais également de l'appropriation des actions collectives signifiantes ("Organisation créatrice d'incertitudes par combinaisons d'actions de plusieurs joueurs : circulations de balle, déplacements des joueurs coordonnés, décalages et variation collective du rythme de jeu »). Selon les préconisations institutionnelles, ces objets de savoirs s'actualisent aussi dans la compétence méthodologique («Savoir utiliser différentes démarches pour apprendre »).

Les critères d'évaluation qui en résultent sont ainsi liés aux configurations collectives (sur laquelle nous nous centrons dans cet article) et aux conduites motrices individuelles (que nous n'étudions pas dans le cadre de cet article). Ils concernent l'observation spécifique de la circulation du ballon et des joueurs (Chateau, 2004) et l'analyse des séquences de jeu amenant à un tir orienté vers la cible adverse, à partir des indicateurs de jeu collectif suivants :

le lieu de la récupération (basse, médiane, haute) ;

la modalité de la récupération (en avant de l'Espace de Jeu Effectif (EJE), au milieu de l'EJE, à la périphérie de l'EJE, en arrière de l'EJE) ;

- le choix de jeu adopté (poursuite du jeu par un tir, une conduite de balle, une passe courte au sol, une passe longue).

Dans l'évaluation au bac, il est à noter que dans les APSA individuelles la performance est appréciée en tenant compte du projet de l'élève et de ce qui a été énoncé avant l'épreuve. Si l'on poursuit cette idée un peu plus loin, pour les sports collectifs, on pourrait mettre en relation le projet tactique collectif avec la réalisation effective sur le terrain. L'analyse de l'évolution des configurations de jeu constitue aussi un outil d'apprentissage 
eJRIEPS Hors série n¹ Décembre 2015

et d'évaluation des progrès des élèves relatif aux productions d'actions collectives et sociales telles qu'instituées par les programmes de la discipline.

\section{Enseigner le football à l'école : une entrée par les apprentissages tactiques collectifs}

Si l'on considère le système enseignement / apprentissage comme un tout, alors l'enseignement par la compréhension et l'apprentissage au travers de la compréhension sont fortement interdépendants. Dans ces approches, ce sont le jeu, l'activité du joueur mais aussi le débat d'idées qui sont mis en relation ; la verbalisation facilite la réflexion, la compréhension et l'observation, ceux-ci fournissant une base de données pour échanger avec les autres joueurs. Si nous voulons suivre cette logique un peu plus loin, nous devrions arriver à la conclusion qu'un objectif majeur en EPS devrait être le développement « d'apprenants réflexifs ".

Quel mode d'entrée l'enseignant d'EPS peut-il alors privilégier dans son contexte d'enseignement, afin de préserver ce qui semble légitime culturellement ? L'enseignant est amené à faire des choix d'objets de savoir culturellement significatifs de l'APSA pour répondre aux objectifs scolaires. Pour sélectionner ces objets de savoir, il convient de ne pas se focaliser uniquement sur l'APSA en tant que pratique sociale, mais plutôt de se centrer sur l'activité de l'élève en train de vivre une expérience motrice signifiante.

2. 1. Une entrée par des situations de jeux à effectif réduit L'utilisation des situations de jeux à effectif réduit permet aux élèves d'être confrontés très fréquemment : à la manipulation du ballon, au duel pour la conquête ou la conservation du ballon, à la possibilité d'atteindre la zone de marque et tenter le tir. Ce mode d'entrée permet de mettre en activité les élèves dans des situations réelles de jeu. En effet, cellesci doivent conserver les éléments incontournables constitutifs des sports collectifs : une aire de jeu délimitée, la présence de cibles, la présence de partenaires et d'adversaires, des équipes identifiées et si possible un rapport de force équilibré (du moins au départ). Cette entrée nécessite également la mise en place et la compréhension par tous des règles spécifiques du football ou de formes de pratiques scolaires élaborées par l'enseignant en EPS. II ne peut avoir de jeu sans mise en place d'un code commun, de règles du jeu simples mais comprises par tous. L'objectif est de transformer leurs représentations initiales liées au football et passer, pour certains, de joueurs valorisant l'affrontement et l'exploit individuel, à la pratique raisonnée et collective du football en milieu scolaire. 


\section{eJRIEPS Hors série n¹ Décembre 2015}

Le choix des situations de jeux à effectif réduit, sous forme de situations d'apprentissage, doit être lié aux objets de savoir retenus par l'enseignant dans le projet de cycle. Ce choix restreint nécessite l'identification des étapes successives à atteindre par les élèves pour progresser. Ces objets de savoir et les situations de jeu correspondantes permettent à l'enseignant et aux élèves d'identifier clairement les différents points de passage significatifs de la compétence attendue en fin de cycle. C'est dans cette perspective que l'enseignant d'EPS pourrait envisager le réinvestissement des savoirs tactiques collectifs par les élèves lors de situations de match.

2. 2. Un débat d'idées au service d'un apprenant réflexif

Le débat d'idées est une pièce centrale d'une conception constructiviste de l'apprentissage des sports collectifs. II consiste après un match, après retour d'informations chiffrées ou de données d'observation, en un dialogue entre les joueurs destiné à faire évoluer ou non le projet d'action de l'équipe en revenant sur la stratégie prévue et en analysant la tactique appliquée. Le professeur peut intervenir à sa convenance en fonction des objectifs poursuivis. Le débat d'idées donne, ainsi, la possibilité aux élèves de partager des expériences collectives dans le cadre d'un rapport de forces en regard des configurations momentanées du jeu apparues. Mais, offrir aux élèves la meilleure éducation physique possible implique bien évidemment de nombreux autres facteurs. L'un des plus importants est de savoir comment les enseignants enseignent et surtout comment les élèves apprennent. Les chercheurs ont besoin d'approfondir les voies d'apprentissage et de décrire, chez les élèves, les processus cognitifs et la construction de l'expérience collective qui se produisent pendant le jeu. En faisant cela, on pourra mieux comprendre les mécanismes qui influencent les relations entre l'acquisition des connaissances et les performances motrices. Un environnement d'apprentissage efficace crée nécessairement des conditions d'enseignement efficientes. En outre, le niveau de pratique de l'élève dans l'activité football influe sur les interactions et la prise de parole des élèves "spécialistes " (Travert, L’Aoustet \& Griffet, 2005) lors des débats d'idées. En effet, les élèves les plus compétents dans l'activité football ont tendance à prendre le leadership des échanges lors des débats et vouloir imposer leur vision du football.

\section{Un parcours de formation authentique et balisé en football : des étapes clés à atteindre}




\section{eJRIEPS Hors série n¹ Décembre 2015}

Le balisage du parcours de formation de l'élève nécessite l'identification des étapes successives à atteindre pour progresser. Cette identification s'appuie sur des indicateurs de progression précis, lisibles et pertinents. Cette démarche d'enseignement permet à l'enseignant de rendre lisible les apprentissages de l'élève. Pour illustrer cette démarche, nous prendrons l'exemple de la compétence attendue de Niveau 4 au lycée (MEN, 2009 ; 2010), en se centrant sur l'évaluation de la "Pertinence et efficacité de l'organisation collective ".

Pour rappel, il est attendu de l'élève à ce niveau de " mettre en œuvre une organisation offensive capable de faire évoluer le rapport de force en sa faveur par une occupation permanente de l'espace de jeu (écartement et étagement), face à une défense qui se replie collectivement pour défendre sa cible ou récupérer la balle». Afin d'identifier les progrès des élèves au niveau de leurs productions d'actions collectives, nous nous baserons sur l'analyse des configurations de jeu amenant à un tir orienté vers la cible adverse (tableau II).

Tableau II. Des étapes clés à atteindre pour acquérir la compétence attendue de niveau 4.

\begin{tabular}{|c|c|c|c|}
\hline Etapes & Objectifs & Ce qu'il faut savoir faire & $\begin{array}{c}\text { Indicateurs de progression: } \\
\text { configurations de jeu }\end{array}$ \\
\hline 1 & $\begin{array}{l}\text { Progresser rapidement } \\
\text { vers la cible adverse }\end{array}$ & $\begin{array}{l}\text { - Pressing des attaquants } \\
\text { - Utilisation préférentielle du } \\
\text { couloir de jeu direct } \\
\text { - Appels en profondeur } \\
\text { privilégiés }\end{array}$ & $\begin{array}{l}\text { Lieu de la récupération : haute } \\
\text { Modalité de la récupération : en } \\
\text { avant de l'EJE } \\
\text { Choix de jeu adopté : conduite ou } \\
\text { passe courte au sol }\end{array}$ \\
\hline 2 & $\begin{array}{l}\text { Contourner et } \\
\text { déséquilibrer la défense } \\
\text { adverse }\end{array}$ & $\begin{array}{l}\text { - Utilisation des couloirs } \\
\text { latéraux } \\
\text { - Occupation de l'espace de } \\
\text { jeu au niveau latéral } \\
\text { (écartement) }\end{array}$ & $\begin{array}{l}\text { Lieu de la récupération : médiane } \\
\text { Modalité de la récupération : à la } \\
\text { périphérie de l’EJE } \\
\text { Choix de jeu adopté : passe } \\
\text { courte au sol }\end{array}$ \\
\hline 3 & $\begin{array}{l}\text { Coordonner et combiner } \\
\text { ses actions à plusieurs au } \\
\text { moment de la } \\
\text { récupération du ballon }\end{array}$ & $\begin{array}{l}\text { - Continuité du jeu assurée de } \\
\text { la récupération au tir } \\
\text { - Occupation de l'espace de } \\
\text { jeu au niveau latéral et } \\
\text { longitudinal (étagement) }\end{array}$ & $\begin{array}{l}\text { Lieu de la récupération : basse } \\
\text { Modalité de la récupération : en } \\
\text { arrière de l'EJE } \\
\text { Choix de jeu adopté : passe } \\
\text { courte au sol ou passe longue }\end{array}$ \\
\hline
\end{tabular}

Nous allons présenter dans les images qui suivent quelques moments clés du jeu que l'on peut considérer comme des phases-méres dans les jeux à effectif réduit car elles contiennent la logique d'exploitation des situations de déséquilibre, qu'elle tend à faire s'enchaîner (Deleplace, 1994). 
eJRIEPS Hors série n¹ Décembre 2015

Capture d'écran étape 1 : Progresser rapidement vers la cible adverse en 6 contre 6

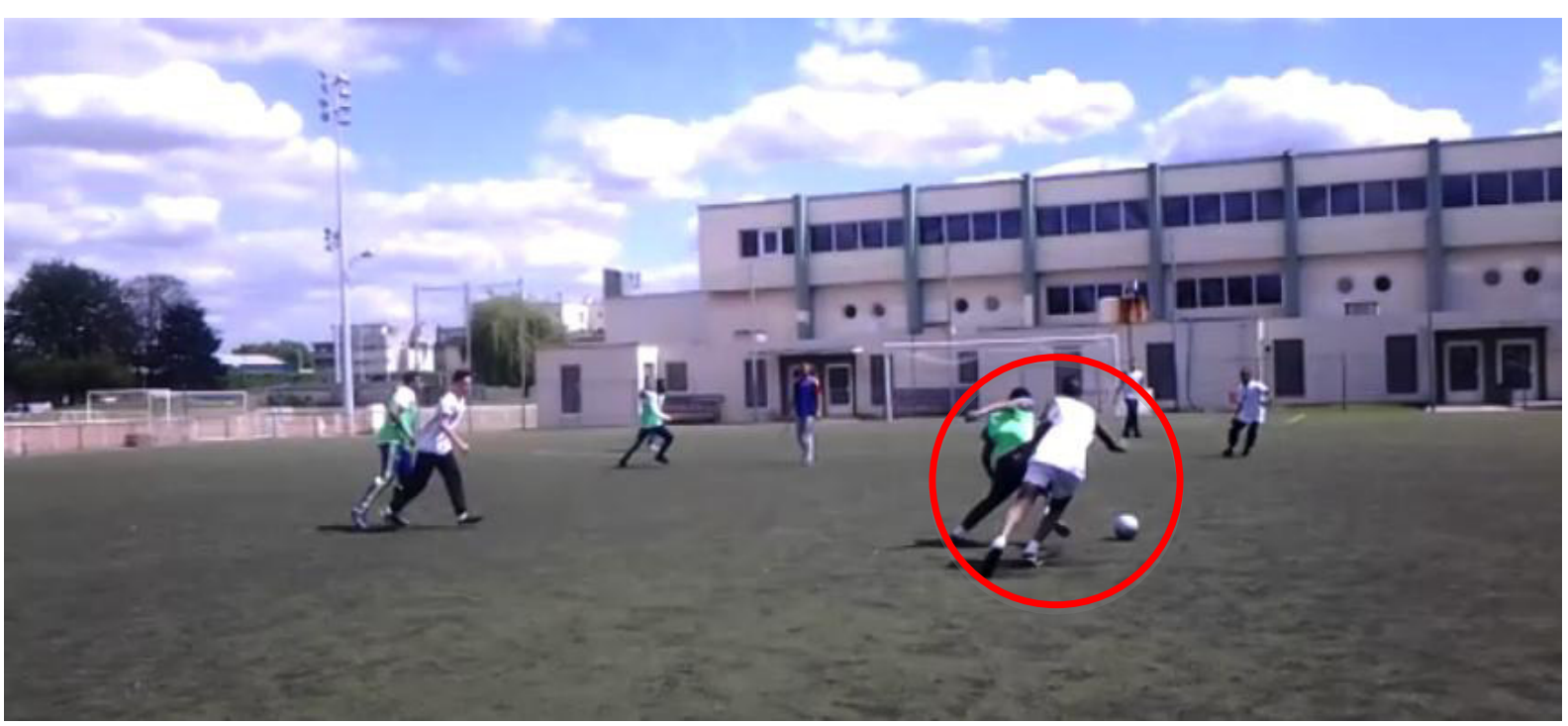

Récupération haute en avant de l'EJE par le joueur en vert

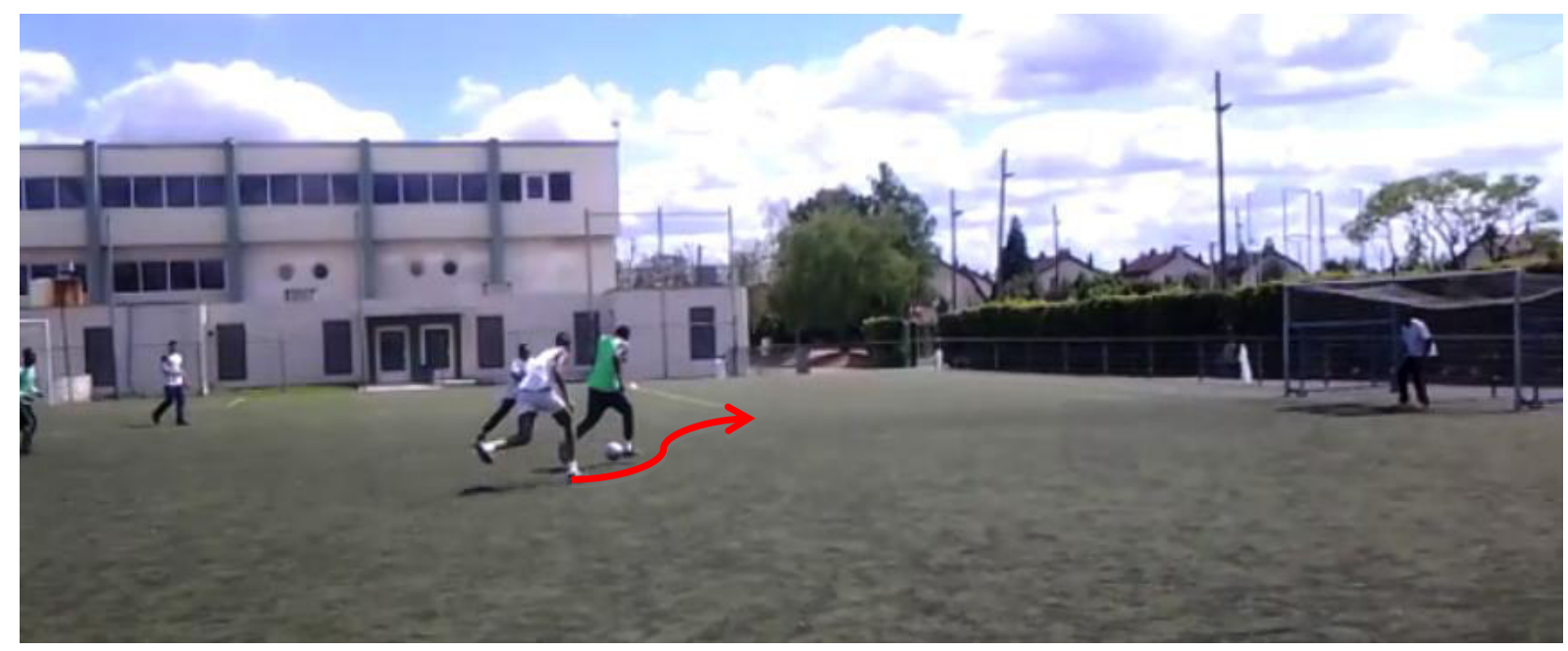

Choix de jeu adopté après la récupération du ballon : conduite dribble 
eJRIEPS Hors série $n^{\circ} 1$ Décembre 2015

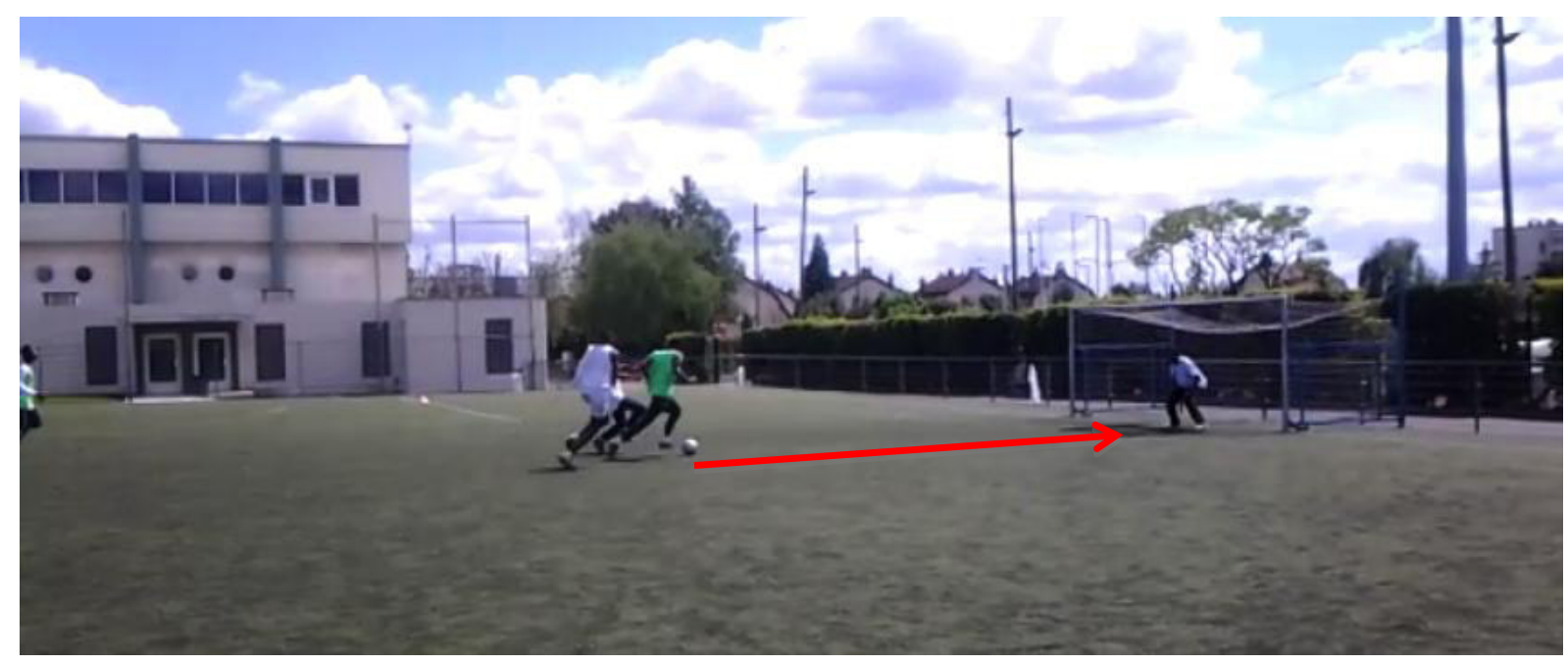

Utilisation du couloir de jeu direct

Capture d'écran étape 2 : Contourner et déséquilibrer la défense adverse

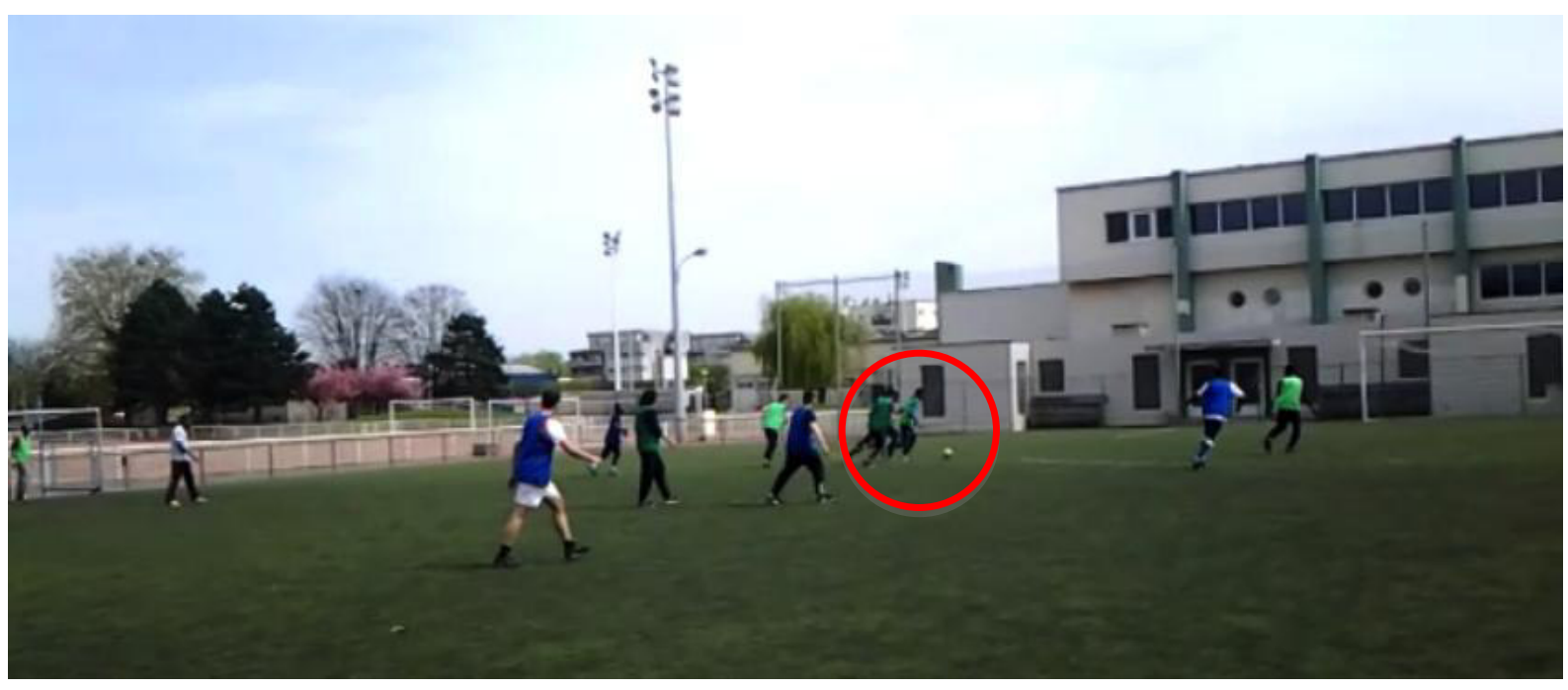

Récupération médiane à la périphérie de l'EJE

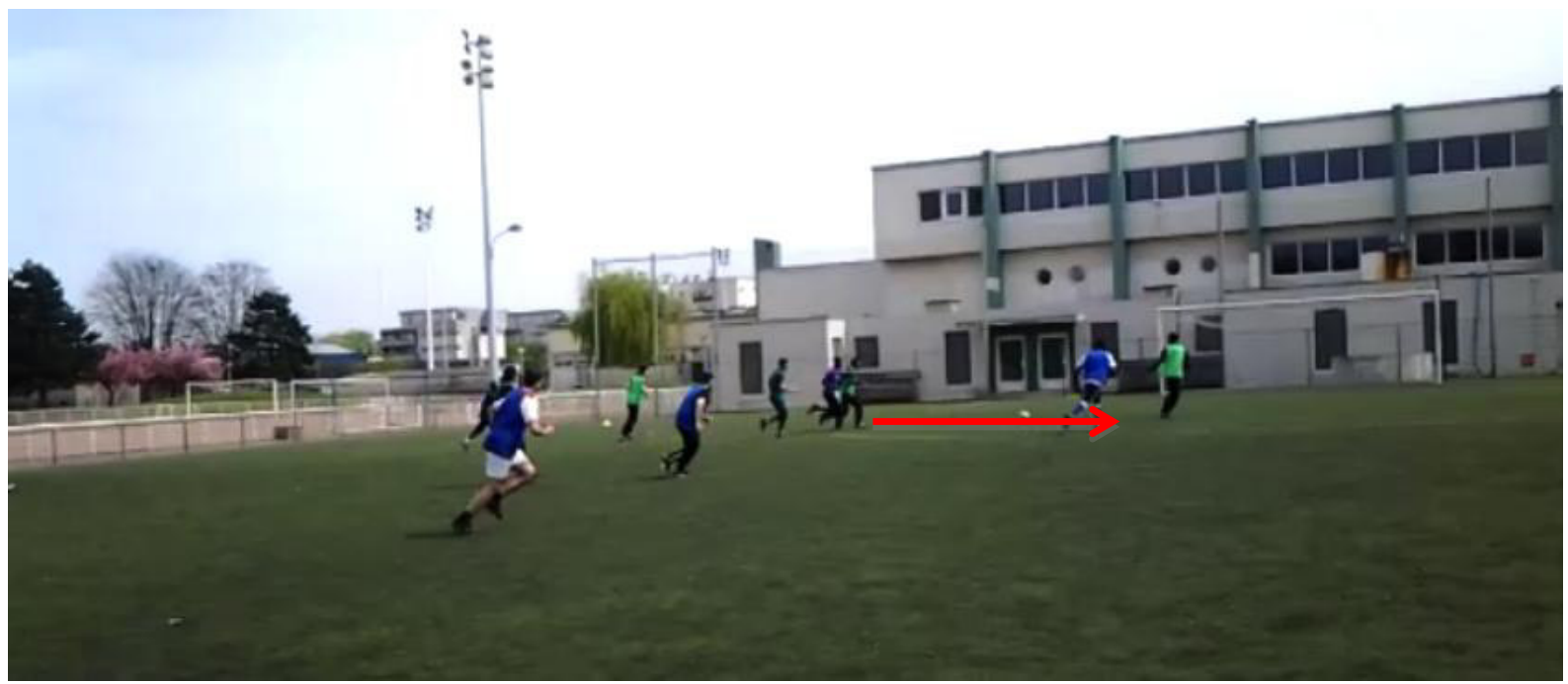


eJRIEPS Hors série n¹ Décembre 2015

Choix de jeu adopté après la récupération du ballon : passe courte pour un partenaire

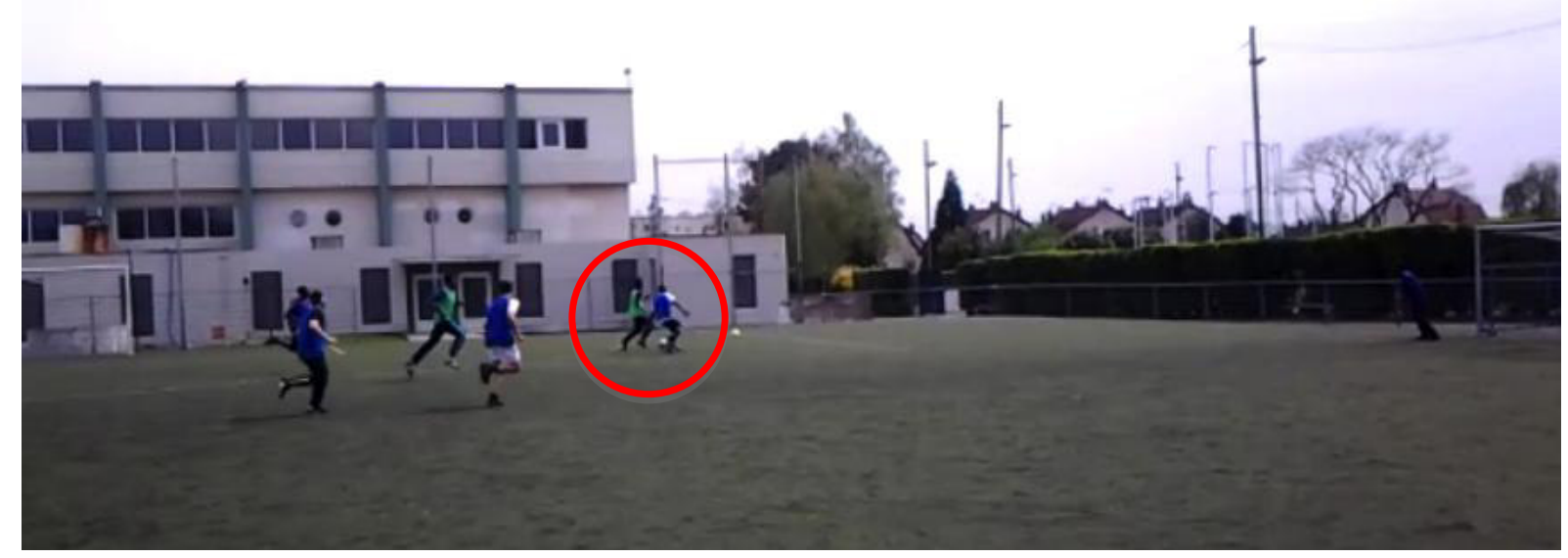

Utilisation des couloirs latéraux

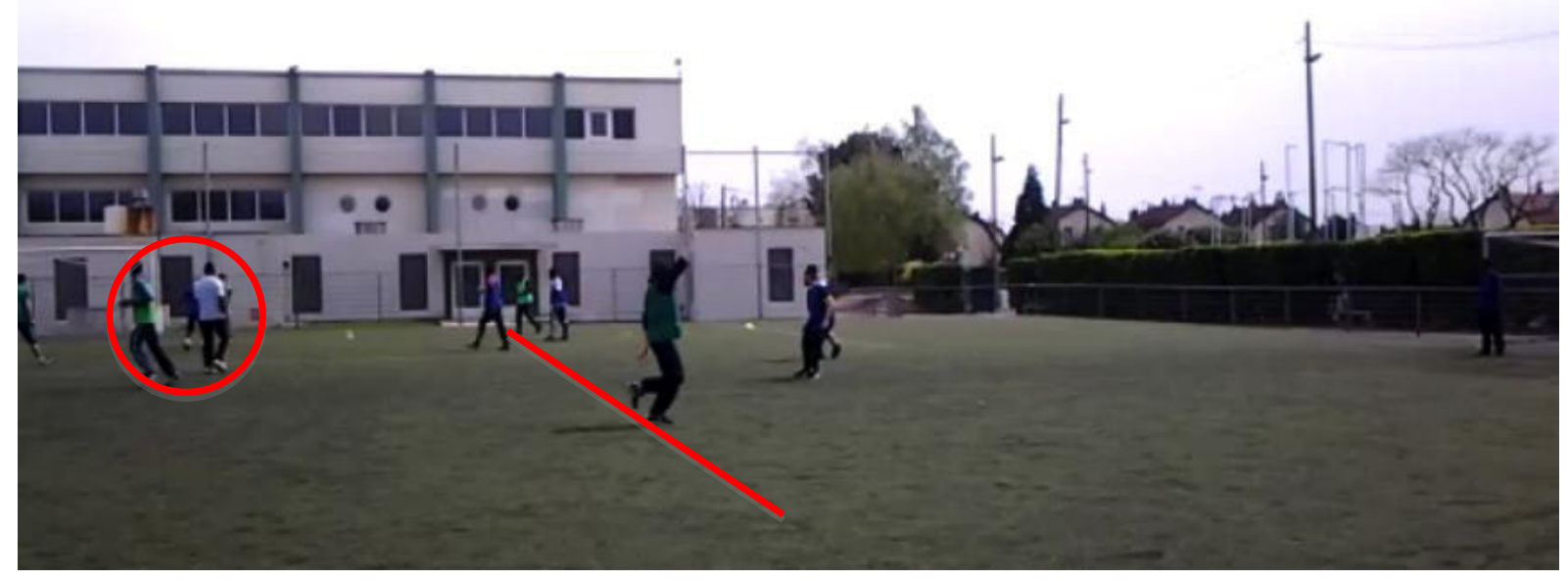

Occupation de l'espace au niveau latéral : écartement du jeu

Capture d'écran étape 3 : Coordonner et combiner ses actions à plusieurs au moment de la récupération du ballon 
eJRIEPS Hors série n¹ Décembre 2015

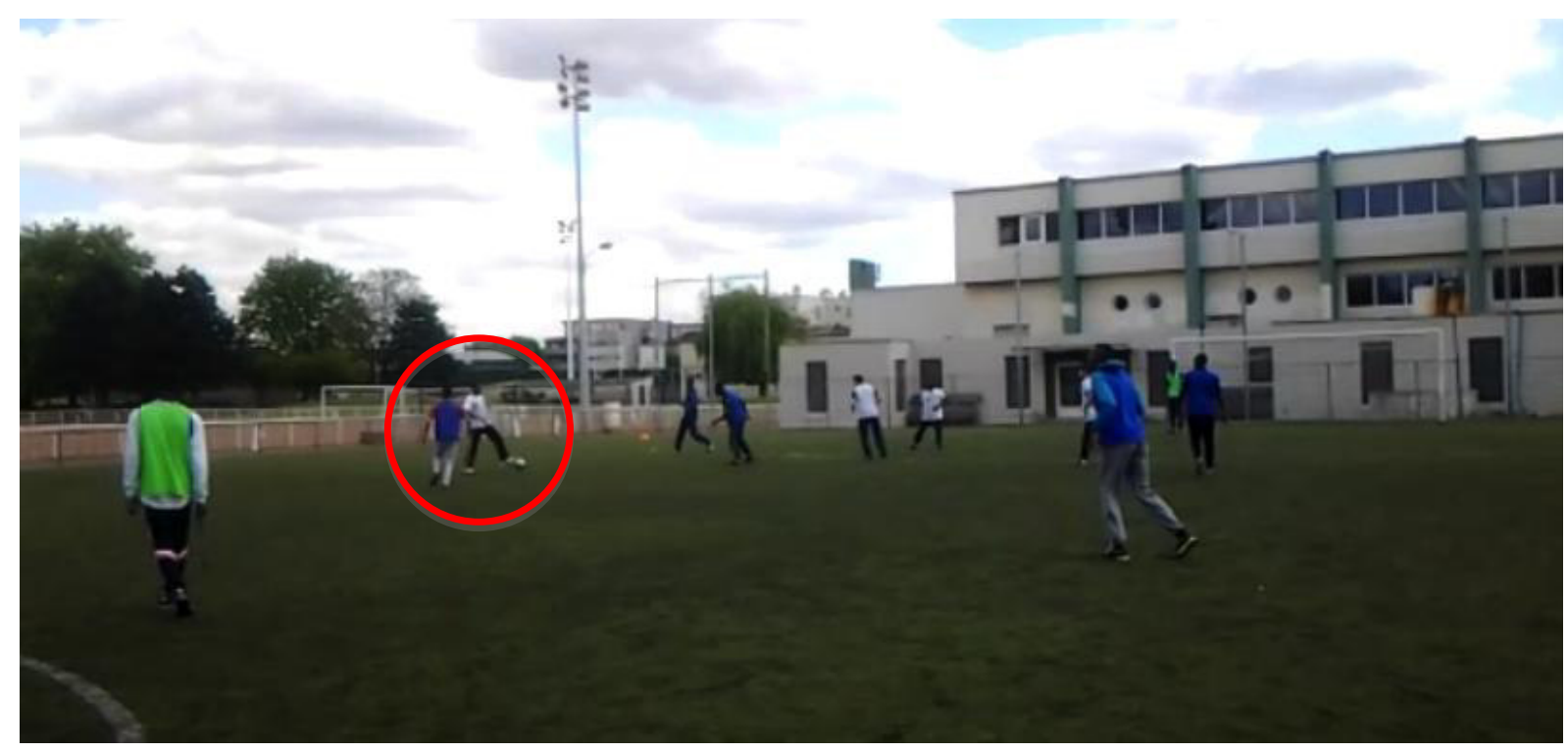

Récupération basse en arrière de l'EJE

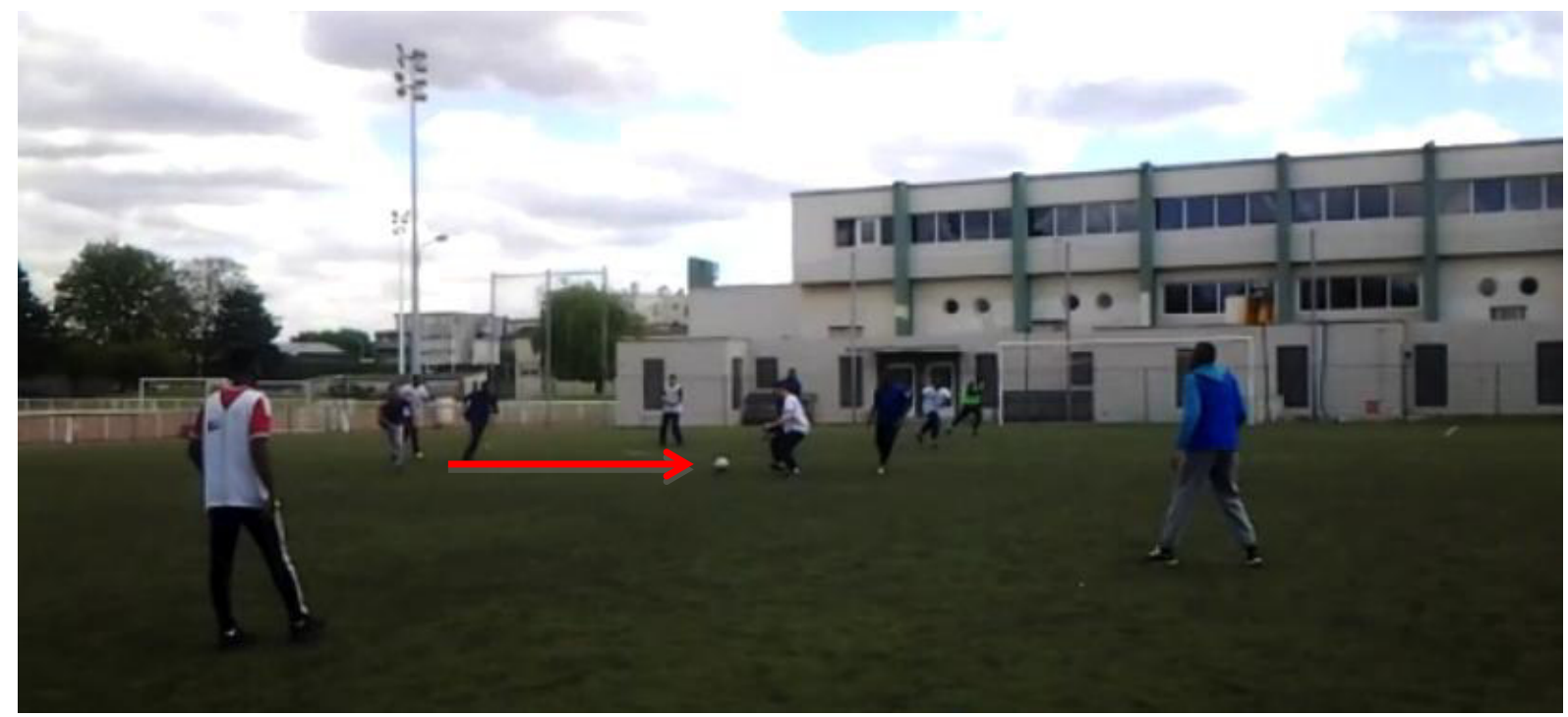

Choix de jeu adopté après la récupération du ballon : passe courte pour un partenaire 


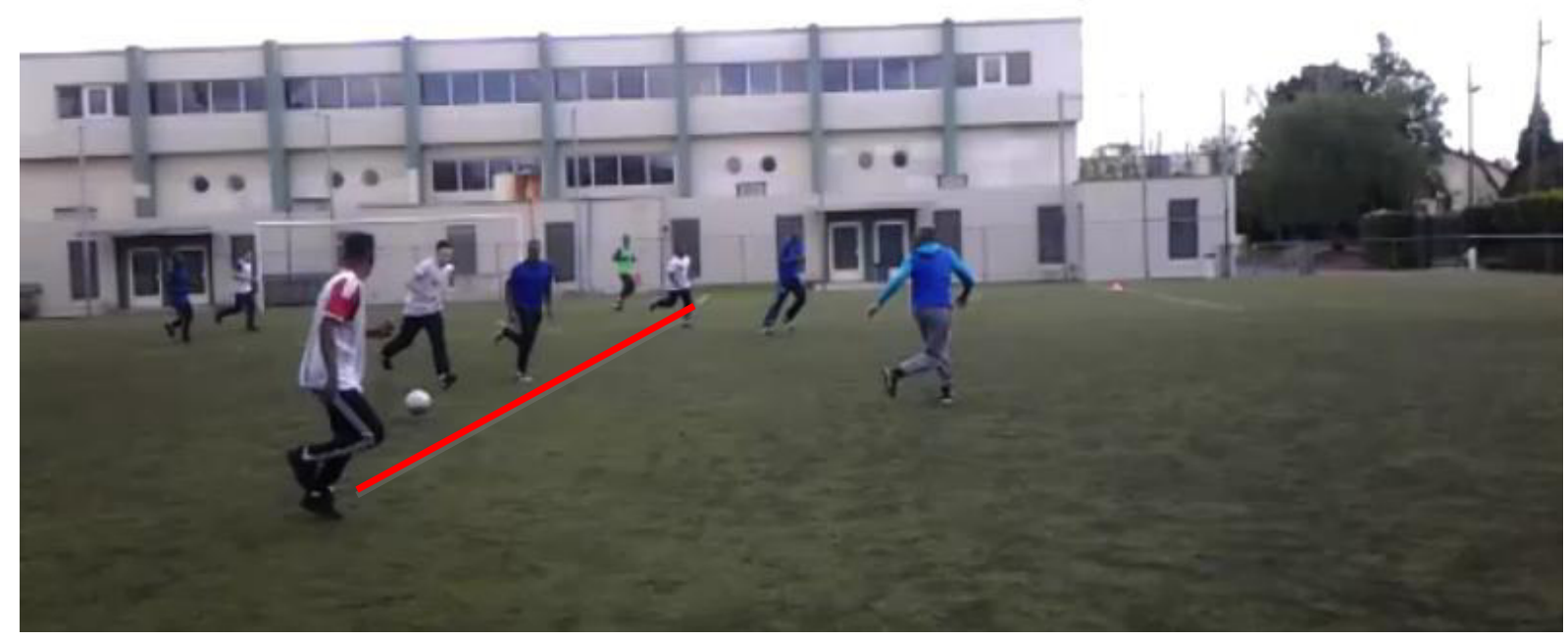

Occupation de l'espace au niveau latéral : écartement du jeu

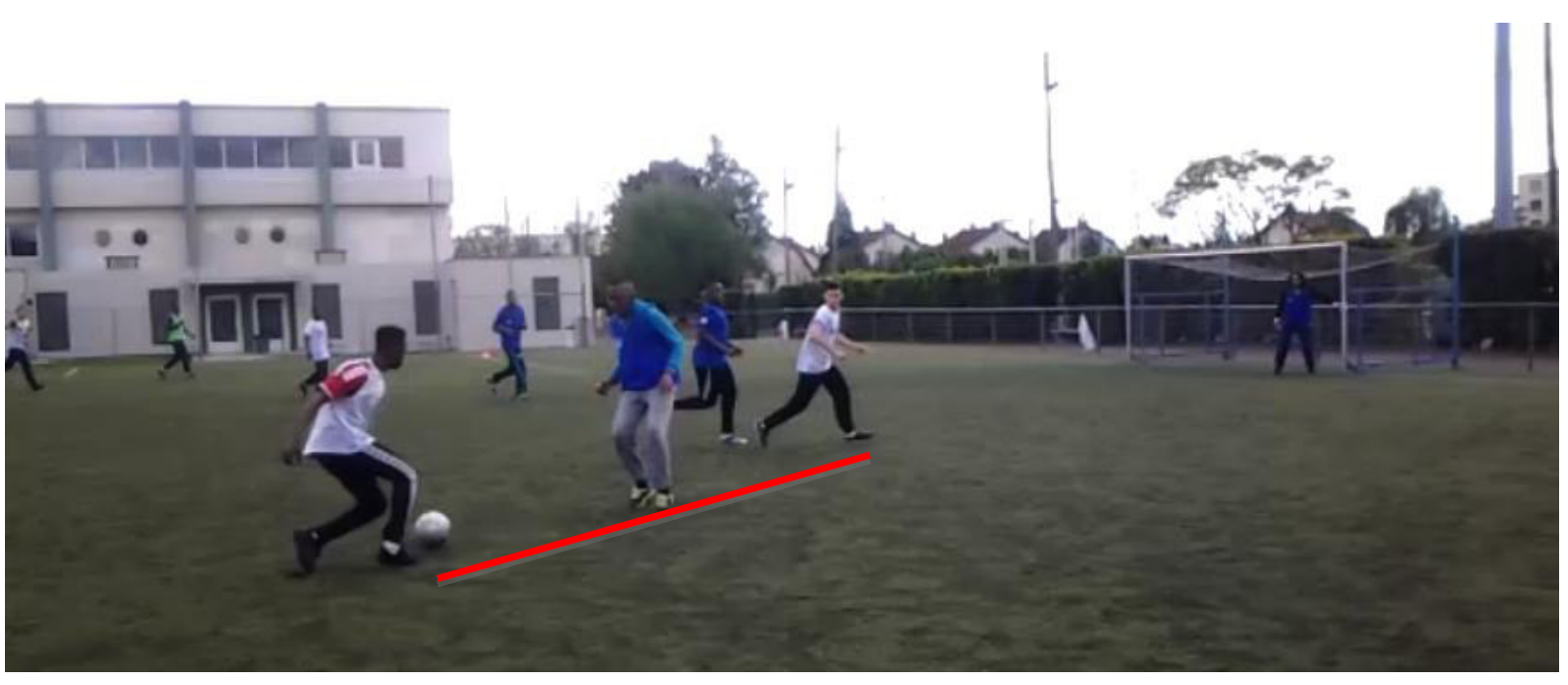

Occupation de l'espace au niveau longitudinal : étagement du jeu

Ces images illustrent bien les différents emplacements de récupération de la balle, des choix et des configurations du jeu qui en découlent. Les phases-mères du jeu constituent, ici, une situation de rupture, un lieu d'apparition de déséquilibres à l'origine des attaques. Cependant il n'est pas question de verser dans le tout cognitif ; il s'agit seulement, dans la gestion des rapports d'opposition en sport collectif, de viser aussi une automatisation partielle des perceptions et des décisions afin de dégager le canal cognitif conscient qui peut alors assurer d'autres opérations. Par apprentissage, cela consiste à faire exécuter, sauf alerte, au niveau de processus cognitifs infra-conscients traités en tâche de fond, un certain nombre de tâches pour offrir la possibilité de faire fonctionner d'autres programmes en même temps. L'évolution s'opère d'un débutant encombré par une 


\section{eJRIEPS Hors série n¹ Décembre 2015}

multitude d'informations vers le joueur de bon niveau qui ne relève que les quelques indices pertinents, voire qui apporte une réponse sans apparemment vraiment avoir relevé d'indice. Lors de ces opérations, la part des processus conscients diminue au profit du traitement d'autres informations. De la sorte, le joueur se bâtit un fonctionnement autonome et une expérience signifiante à partir d'une base de données constituée par des savoirs et des pratiques acquises au contact du jeu.

Bouthier et Savoyant (1984) soutiennent que dans toute action individuelle on doit distinguer, à la suite de Galpérine (1980) des opérations d'organisation (celles qui assurent le repérage de la situation et amènent à une décision) en rapport avec la base d'orientation du sujet (elles précédent les autres d'où la minoration des apprentissages technicistes) et des opérations d'exécution. Pour Galpérine (1965), la structure interne de l'action est complexe car outre un but et un mobile, elle suppose la présence d'un objet, et aussi, chez le sujet, d'une base d'orientation. Cette dernière comprend l'ensemble de ses connaissances disponibles, à propos de l'action elle-même aussi bien que des conditions dans lesquelles elle est accomplie. Enfin, toute action est composée d'un ensemble d'opérations. C'est l'ordre dans lequel celles-ci sont exécutées qui constitue le mécanisme de l'accomplissement de l'action. Quant à l'analyse fonctionnelle, elle distingue dans l'action trois parties : orientation, exécution et contrôle.

\section{De l'APSA aux formes de pratiques scolaires du football en EPS}

L'enseignement de l'EPS ne peut se réduire à l'« étude » d'APSA. II convient également de s'intéresser aux "formes de l'étude » (Chevallard, 1997), c'est-à-dire aux modalités didactiques et pédagogiques adaptées à l'étude. En ce sens, il s'agit de faire vivre aux élèves l'épaisseur culturelle des activités physiques sportive et artistique, finalité soulignée dans les programmes d'EPS (MEN, 2008; 2009 ; 2010), tout en transposant dans le contexte scolaire les savoirs tels qu'ils existent initialement dans la société. Poursuivre cette ambition revient finalement à s'interroger sur les signes distinctifs qui constituent le fond culturel de chaque activité. Par ailleurs, cela oblige les enseignants à proposer des formes de pratiques scolaires qui s'émancipent de la forme de pratique sociale de référence, tout en étant révélatrices de la compétence attendue.

En EPS, de nombreux travaux (particulièrement développés dans le domaine professionnel) s'intéressent à l'élaboration de formes de pratiques scolaires dont l'enjeu est de confronter les élèves à des pratiques culturelles signifiantes mais adaptées au 


\section{eJRIEPS Hors série n¹ Décembre 2015}

contexte scolaire (C.E.D.R.E., 2007 ; Dhellemmes, 2006 ; Mascret, 2009 ; Mascret \& Dhellemmes, 2011 ; Dietsch, 2015).

L'élaboration d'une forme de pratique scolaire nécessite une contextualisation de l'analyse des activités des élèves et des pratiques sociales et culturelles qui lui donnent sens. Toutefois, cette nécessaire contextualisation ne doit pas amener l'enseignant à sur-ajuster cette forme de pratique aux caractéristiques des élèves. En effet, l'intérêt de proposer une forme de pratique scolaire en EPS est d'offrir aux élèves une expérience motrice et socialisatrice différente et signifiante par rapport à leur socialisation familiale et vis-à-vis de la pratique sociale. L'approche par les jeux à effectif réduit ou le futsal, contrairement à l'approche traditionnelle, propose d'apprendre à jouer en situation réelle en s'appuyant sur la motricité habituelle plutôt que sur la primauté de prérequis basés sur des savoir-faire techniques. En outre, cette approche des jeux permet aux élèves de découvrir, par euxmêmes, ce qu'il faut faire dans le jeu. Cette découverte guidée qui aide les joueurs à résoudre les problèmes qui se posent dans le jeu, peut constituer, aussi, une grande partie du plaisir d'apprendre.

Dans le cadre de la mise en œuvre d'une forme de pratique scolaire du football, l'expérience aura du sens pour l'élève s'il s'intéresse à l'action collective, s'il y voit un intérêt personnel, si les élèves sentent qu'ils dépendent les uns des autres et adoptent des comportements qui le permettent, et enfin si les règles mises en place amènent une plus value dans leur pratique. L'important est que les évolutions règlementaires apportées en EPS et en football amènent l'élève à vivre une réelle expérience de joueur de football, et que les conditions de pratique, mises en œuvre par l'enseignant, lui permettent de devenir plus efficace dans cette APSA.

Enfin, nous considérons que l'enseignement des sports collectifs et notamment du football ne peut faire l'économie d'une réflexion sur le «temps de concertation » (MEN, 2009 ; 2010) entre les séquences de jeu, utiles pour permettre aux joueurs d'une même équipe d'ajuster leurs organisations collectives en fonction du jeu adverse, mais également dans l'optique de viser une amélioration positive dans la tenue de rôles sociaux. Cette démarche propose ainsi une co-construction collective des savoirs sur le jeu et sur les rôles sociaux, à travers un débat d'idées ou un temps de concertation en différentes phases : une séquence de jeu ; un temps de débats d'idées entre les équipes; un bilan des débats effectués par l'enseignant (Dietsch, Brière \& Wane, sous presse).

Comme dans bien des cas, l'expérience singulière du joueur n'est cependant pas une entité uniforme et les distinctions à opérer en son sein sont des distinctions utiles pour 


\section{eJRIEPS Hors série n¹ Décembre 2015}

l'analyse. L'aide à la compréhension de la construction de cette expérience et son orientation possible apparaît aujourd'hui comme une voie majeure de développement de la formation du joueur. Ce sont sans doute moins les connaissances produites par l'expérience qui importent que la manière dont elles se construisent et se transforment dans la dialectique de l'affrontement. Aussi chaque régression apparente, chaque évolution ou progression constituent à la fois le soubassement et le moyen du progrès à venir. L'expérience du joueur représente de la sorte le produit des interactions entre le sujet et les différents aspects du jeu, ces derniers ayant chacun leurs règles de fonctionnement propres en rapport avec la trame dynamique du jeu. Au fil du temps, ils sont reconnus sous forme de configurations qui permettent de lire et d'interpréter la propension l'action en cours. Cela permet de comprendre comment un espace topologique organisé autour de la position des joueurs et du ballon est traversé et transformé par des vitesses, des accélérations et décélérations ainsi que des changements de direction pour devenir un espace dynamique que le joueur expérimenté peut décoder. Enfin, cette expérience, obligatoirement inscrite dans l'histoire du sujet, consiste à répéter dans le temps et de façon cyclique la mise en relation entre vécu du joueur, construction de connaissances et partage de cette expérience dans la communauté des joueurs qu'est l'équipe.

\section{Conclusion : faire vivre une expérience collective de joueur de football}

Enseigner le football à l'école rejoint l'idée d'acculturation, ce qui revient à faire vivre à certains élèves des expériences motrices et sociologiques différentes de leurs modèles culturels initiaux (Redfield, Linton \& Herskovits, 1936).

Sur ce point, l'école et l'EPS jouent un rôle décisif en proposant et en confrontant les élèves à une expérience socialisatrice différente. Nous postulons que les formes de pratiques scolaires doivent permettre à tous les élèves d'accéder au fondement culturel des activités (Berchebru, Meunier, \& Gréhaigne, 2009). Si les contraintes inhérentes à l'enseignement en milieu scolaire doivent être nécessairement prise en compte, elles n'ont toutefois pas lieu d'entraver l'accès à une culture physique. Par conséquent, le vécu d'expériences corporelles doit être considéré comme le fer de lance de l'enseignement en EPS

Le modèle de fonctionnement et de développement du joueur de sport collectif qui vient d'être esquissé met l'accent sur la pluralité des processus et sur la nature des relations qu'ils entretiennent dans le fonctionnement de l'élève. Cette vision du joueur semble 


\section{eJRIEPS Hors série n¹ Décembre 2015}

universel dans le mesure où le répertoire des processus est le même pour tous les sujets. Elle est différentielle dans la mesure où la hiérarchie entre les éléments du système de variables et de valeurs est changeante selon les affrontements, les équipes et / ou selon les individus. Ce type de fonctionnement fait de l'interaction entre ces processus et des modes de traitement une source du développement du joueur. Cette approche pluraliste admet, par construction, qu'un même problème puisse être résolu par des processus différents. Elle ne situe pas les différences entre joueurs seulement dans les propriétés des situations mais aussi dans les modes de représentation et de traitement qu'elle sollicite.

En définitive, les notions de disponibilité, d'interactions, et de mises en relation constituent, nous semblent-ils, des éléments décisifs pour appréhender le jeu et le développement du joueur. Les sports collectifs à l'école restent un formidable outil pour travailler en groupe et apprendre à gérer des trajets et des trajectoires de mobiles dans des conditions d'urgence décisionnelle.

\section{Bibliographie}

Berchebru, M., Meunier, J.-N., \& Gréhaigne J.-F. (2009). L'utilisation de films vidéo illustrant des configurations prototypiques du jeu et de débats d'idées dans la didactique du football en milieu scolaire. In J.-F. Gréhaigne, (Ed.) Autour du temps. Espaces, apprentissages, projets dans les sports collectifs (pp. 103-126). Besançon : Presses de l'Université de Franche-Comté.

Bouthier, D., \& Savoyant, A. (1984). A contribution to the learning of a collective action: the counter-attack in rugby. International Journal of Sport Psychology, 15 (1), 25-34.

Caillé, A. (2008). Au-delà de l'intérêt. Revue du Mauss semestrielle, 31, 95-115.

C.E.D.R.E. (2007). Pour une culture scolaire des APSA en EPS. Revue EPS, 328, 61-68.

Chateau, L. (2004). Analyse sémiotique des configurations prototypiques offensives chez les débutants en football. Mémoire de DEA en Science du langage, didactique, sémiotique. Université de FrancheComté.

Chevallard Y. (1997). Les savoirs enseignés et leurs formes scolaires de transmission : un point de vue didactique. Skholê (7), 45-64.

Deleplace, R. (1994). La notion de matrice d'action pour les actions motrices complexes. In D. Bouthier \& J. Griffet (Eds.) Représentation et action en activité physique et sportive (pp. 25-42). Université de Paris-Orsay.

Dhellemmes R. (2006). Formes de pratique scolaires d'APSA : l'émotion, l'espace et... le reste ! Les Cahiers du CEDRE $n^{\circ} 5$, 35-45.

Dietsch, G. (2015). Approche technologique et forme de pratique scolaire du football en milieu difficile : le modèle du « futsal ». eJRIEPS, 35, 60-85.

Dietsch, G., Brière-Guenoun, F \& Wane, T-C. (2015). Forme de pratique scolaire du football en EPS, débats d'idées et apprentissages en milieu difficile. Recherches en Education, 23, 117-131.

Dugrand M. (1989) Le football : de la transparence à la complexité. Paris : PUF.

Galperine, P. (1965). Les principaux résultats des recherches sur le problème de la formation des actions et des concepts intellectuels ». Moscou.

Galperine, P. (1980). Essai sur la formation par étapes des actions et des concepts. In N.F. Talyzina (Ed.) De l'enseignement programmé à la programmation de la connaissance (pp.167-183). Lille : Presses Universitaires.

Gréhaigne, J.F. (Ed.). (2007). Configurations du jeu, débat d'idées et apprentissage des sports collectifs. Besançon : Presses de l'Université de Franche-Comté. 


\section{eJRIEPS Hors série n¹ Décembre 2015}

Gréhaigne, J.F., Caty, D., \& Marle, P. (2004). L'apport de la notion de configuration du jeu à la didactique des sports collectifs. In G. Carlier (Ed.), Si l'on parlait du plaisir d'enseigner l'éducation physique (pp. 167-179). Montpellier : AFRAPS.

Grün, L. (2012). L'enseignement scolaire du football. Un développement contrarié (1890 à nos jours). In C. Ottogalli-Mazzacavallo \& P. Liotard (Eds.). L'éducation du corps à l'école. Mouvements, normes et pédagogies. 1881-2011 (pp. 281-293). Ed. AFRAPS.

Marle, P., \& Duprat, E. (2014). Au football, la règle du hors-jeu a-t-elle encore un sens ? eJRIEPS, 32, 7286.

Marle, P. Zerai, Z., \& Gréhaigne, J.-F., (2011). Des données, des indices pour observer, évaluer... et donner du sens. In J.-F. Gréhaigne (Ed.) Des signes au sens. Le jeu, les indices, les postures et les apprentissages dans les sports collectifs à l'école (pp. 159-174). Besançon: Presses de I'Université de Franche-Comté.

Mascret, N. (2009). Référence culturelle et formation des enseignants d'Education Physique et Sportive, Travail et Formation en Education, 3, [En ligne], mis en ligne le 18 décembre 2009. URL: http://tfe.revues.org/index870.html.

Mascret, N., \& Dhellemmes, R. (2011). Culture sportive et culture scolaire des APSA. In M. Travert \& N. Mascret (Eds.). La culture sportive (p. 99-115). Paris, Éditions EP\&S.

MINISTĖRE DE L'ÉDUCATION NATIONALE (2008). Programmes d'EPS pour les classes de collège. B.O spécial $n^{\circ} 6$ du 28 août.

MINISTĖRE DE L'ÉDUCATION NATIONALE (2009). Programmes d'EPS pour les classes de lycée professionnel. B.O. spécial n² du 19 février.

MINISTĖRE DE L'ÉDUCATION NATIONALE (2010). Programmes d'EPS pour les classes de lycée général et technologique. BO spécial $n^{\circ} 4$ du 29 avril.

Redfield, R., Linton, R., \& Herskovits, M. J. (1936). Memorandum for the study of acculturation. American anthropologist, 38(1), 149-152.

Travert, M. (1997). Le "football de pied d'immeuble" : une pratique singulière au cœur d'une cité populaire, Ethnologie française, $\mathrm{n}^{\circ} \mathrm{XXVII}, 188-196$.

Travert, M., L'Aoustet, O., \& Griffet, J. (2005). Les élèves et les sports. Revue EPS, 315, 29-32. 